\title{
Inbalt des fünften Bandes (Teil I)
}

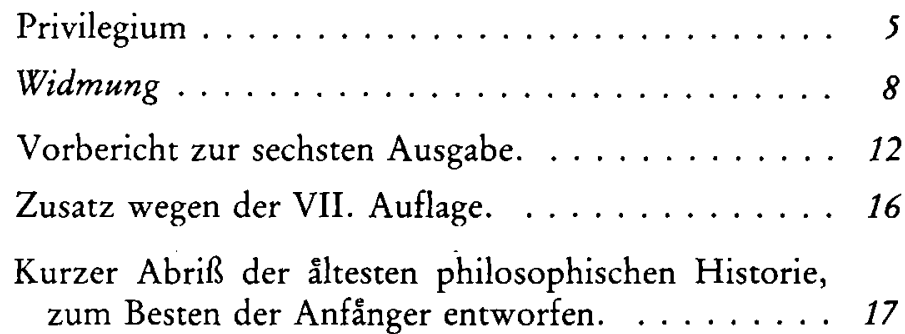

Der åltesten philosophischen Historie I. Abschnitt.

Von der orientalischen oder asiatischen Philosophie.

Das I. Hauptstůck.

Von der Weltweisheit der ersten Menschen. Vor der Súndfluth. . . . . . . . . . . . . . . . . . . 19

Das II. Hauptstúck.

Von der chinesischen Weltweisheit. . . . . . . 23

Das III. Hauptstůck.

Von der chaldåischen Philosophie. . . . . . . . 30

Das IV. Hauptstůck.

Von der Weltweisheit der Hebråer. . . . . . . . . 35

Das V. Hauptstůck.

Von der Weltweisheit der alten Perser. . . . . . . . 40

Das VI. Hauptstůck.

Von der indianischen Weltweisheit. . . . . . . 45

Das VII. Hauptstůck.

Von der Weltweisheit der Araber und Sabåer. . . . 50 
Das VIII. Hauptstůck.

Von der Weltweisheit der alten Phónizier. . . . . . 57

II. Abschnitt.

Von der afrikanischen Volker Weltweisheit.

Das I. Hauptstůck.

Von der ågyptischen Philosophie. . . . . . . . 63

Das II. Hauptstück.

Von der Weltweisheit der Aethiopier und anderer africanischer Volker. . . . . . . . . . . . . . . 72

III. Abschnitt.

Von der Weltweisheit der Europåer.

Das I. Hauptstůck.

Von der Philosophie der Celten und Scythen. . . . . 77 Das II. Hauptstůck.

Von der åltesten Fabelphilosophie der Griechen. . . 84 Das III. Hauptstůck.

Von der Philosophie der sieben Weisen in Griechenland. . . . . . . . . . . . . . . . . 92

Das IV. Hauptstůck.

Von der ionischen Secte der Weltweisen. . . . . . . . 99 Das V. Hauptstuick.

Von der italischen Schule der Weltweisheit. . . . . 105 Das VI. Hauptstůck.

Von der Sokratischen Schule der Weltweisheit. . . . 110

Erste Gründe der Weltweisheit.

Theoretischer Theil.

Einleitung zur Weltweisheit ůberhaupt $\ldots \ldots \ldots 122$ 
Der theoretischen Weltweisheit Erster Theil.

Die Vernunftlehre.

Einleitung zur Vernunftlehre. . . . . . . . . . . . 130

Der Vernunftlehre Erster Theil.

Von den dreyen Kråften des menschlichen Verstandes.

Das I. Hauptstůck.

Von der Empfindungskraft. (De Perceptione et Ideis.) . . . . . . . . . . . . . . . . . . . . . 134

Das II. Hauptstůck.

Von den Erklårungen der Wörter und Sachen. (Definitionibus nominalibus et realibus.) . . . . . . . 141

Das III. Hauptstůck.

Von der Urtheilskraft, und von den Såtzen. (De judicio et propositionibus.) . . . . . . . . . . 146

Das IV. Hauptstůck.

Von dem Unterschiede der Såtze, im Absehen auf die mathematische Lehrart. . . . . . . . . . . . . . 151

Das V. Hauptstúck.

Von der Kraft zu schließen und von den Schlußreden. . . . . . . . . . . . . . . . . 153

Das VI. Hauptstück.

Von den Beweisen und ihren mancherley Arten. . . 167

Der Vernunftlehre zweiter Theil.

Von den Nutzen derselben.

Das I. Hauptstůck.

Von dem Nutzen der Vernunftlehre in Erfindung unbekannter Wahrheiten. . . . . . . . . . . . . . 174

Das II. Hauptstůck.

Von dem Nutzen der Vernunftlehre in dem guten

Vortrage der erfundenen Wahrheiten. ........ 182 
Das III. Hauptstůck.

Von dem Nutzen der Vernunftlehre in Beurtheilung erfundener Wahrheiten und der Schriften, darinn sie vorgetragen wird. . . . . . . . . . . . . . 203

Das IV. Hauptstůck.

Vom Nutzen der Vernunftlehre im Bücherlesen und der Wahl guter Lehrer in Kunnsten und Wissenschaften. . . . . . . . . . . . . . . . . . . 212

Das V. Hauptstůck.

Vom Nutzen der Vernunftlehre im Erklåren, Ueberführen, Widerlegen und Disputiren. . . . . . . . . 217

Der theoretischen Weltweisheit Zweyter Theil.

Die Grundlehre, oder Metaphysik.

Einleitung zur Grundlehre. . . . . . . . . . . . . . . 224

Der Grundlehre I. Abschnitt.

Die Ontologie.

Das I. Hauptstůck.

Von den beyden Hauptgründen der Vernunft. . . 227

Das II. Hauptstůck.

Von einem Dinge ůberhaupt, auch von seinem We-

sen und Daseyn. . . . . . . . . . . . . . . . . . . . . 229

Das III. Hauptstůck.

Von den allgemeinen Beschaffenheiten eines Dinges. 232 Das IV. Hauptstúck.

Von den zusammengesetzen Dingen. . . . . . . 243

Das V. Hauptstück.

Von den einfachen Dingen. . . . . . . . . . . . . . 249

Das VI. Hauptstůck.

Von den Verhåltnissen der Dinge gegen einander. . 256 
Der Grundlehre II. Abschnitt.

Die Weltbetrachtung.

Das I. Hauptstůck.

Von dem Wesen einer Welt und von ihren vornehmsten Eigenschaften. . . . . . . . . . . . . . . 262

Das II. Hauptstůck.

Von dem Wesen und der Natur der Kórper. . . . 271 Das III. Hauptstůck.

Von den Elementen der Körper. . . . . . . . . . . . 279

Das IV. Hauptstůck.

Von dem Natůrlichen und Uebernatưrlichen. . . . . 288 Das V. Hauptstúck.

Von der Vollkommenheit der Welt und vom Laufe der Natur.

Der theoretischen Weltweisheit Dritter Theil.

Die Naturlehre.

Einleitung zur Naturlehre. . . . . . . . . . . . . . . 298

Der Erste Abschnitt.

Von den Ko̊rpern ůberhaupt.

Das I. Hauptstůck.

Von den allgemeinen Eigenschaften der Ko̊rper. . . 300

Das II. Hauptstůck.

Von dem Unterschiede der Kơrper, in Ansehung

ihrer eigenthůmlichen Materie. . . . . . . . . . 306

Das III. Hauptstůck.

Von dem Unterschiede der Ko̊rper, der von fremder

Materie herrủhret. . . . . . . . . . . . . . . . . . 310

Das IV. Hauptstůck.

Von der Bewegung der festen und flüssigen Körper. 318 
Der zweyte Abschnitt.

Vom ganzen Weltgebåude.

Das I. Hauptstůck.

Von den Weltkơrpern ủberhaupt. . . . . . . . . . . . 329

Das II. Hauptstúck.

Von der Sonne. . . . . . . . . . . . . . . . 335

Das III. Hauptstůck.

Von dem Monde und den Planeten. . . . . . . . . . 347

Erster Abschnitt vom Monde. . . . . . . . . . . . . . 347

Zweiter Abschnitt von den Planeten. . . . . . . . . 351

Das IV. Hauptstůck.

Von dem planetischen Weltbaue. ......... 356

Das V. Hauptstůck.

Von den Kometen oder Schwanzsternen. . . . . . . . 365

Das VI. Hauptstůck.

Von den Fixsternen. . . . . . . . . . . . . . . 376

Der dritte Abschnitt.

Von der Erdkugel, ihren Theilen und Verånderungen.

Das I. Hauptstůck.

Von der Erdkugel ůberhaupt. . . . . . . . . . . 381

Das II. Hauptstůck.

Von den vier Jahreszeiten und ihren Witterungen. . 394

Das III. Hauptstuick.

Von der Luft und von den Winden. . . . . . . . . 400

Das IV. Hauptstůck.

Von dem Wasser in Meeren und Flüssen. . . . . . 409

Das V. Hauptstück.

Von den wåsserichten Luftbegebenheiten. (De meteoris aqueis.) . . . . . . . . . . . . 417

Das VI. Hauptstůck.

Von den glănzenden Luftbegebenheiten. (De meteo-

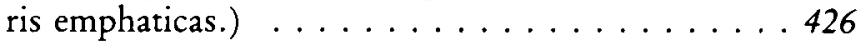


Das VII. Hauptstůck.

Von den feurigen Luftbegebenheiten. (De meteoris igneis.) . . . . . . . . . . . . . . . 435

Das VIII. Hauptstück.

Von der Erde, den Mineralien und Steinen. . . . . . 441

Das IX. Hauptstůck.

Von dem Magneten. . . . . . . . . . . . . . . . . . 448

Das X. Hauptstůck.

Von dem gemeinen und unterirdischen Feuer. . . 454

Anhang zum dritten Abschnitte, oder XI. Capitel.

Von der elektrischen Kraft der Körper . . . . . . . 459

Der vierte Abschnitt.

Von den lebendigen Kòrpern, als Pflanzen und Thieren.

Das I. Hauptstůck.

Von den Pflanzen. . . . . . . . . . . . . 465

Das II. Hauptstůck.

Von den Thieren ůberhaupt. . . . . . . . . . . 476

Das III. Hauptstúck.

Von den Bewegungen und sinnlichen Werkzeugen

der Thiere. . . . . . . . . . . . . . . . 486

Das IV. Hauptstůck.

Von dem menschlichen Ko̊rper. . . . . . . . . . . . . 494 
Der theoretischen Weltweisheit Vierter Theil.

Die Geisterlehre.

Einleitung zur Geisterlehre. . . . . . . . . 508

Der Geisterlehre I. Abschnitt.

Von den Krăften der Seelen und der Geister.

Das I. Hauptstůck.

Von dem wirklichen Daseyn unserer Seelen. . . . . 511

Das II. Hauptstůck.

Von der Empfindungskraft. . . . . . . . . . . . 514

Das III. Hauptstück.

Von der Einbildungskraft und dem Gedåchtnisse. . 518

Das IV. Hauptstúck.

Von dem Verstande, und der Beurtheilungskraft. . 523 Das V. Hauptstúck.

Von der Vernunft und der Erfindungskraft. . . . . 531 Das VI. Hauptstů́ck.

Von der sinnlichen Begierde und den Affecten. . . 538 Das VII. Hauptstůck.

Von dem Willen und der Freyheit. . . . . . . . 546 Das VIII. Hauptstúck.

Von der Herrschaft der Seele über den Leib, und von ihrer Verknüpfung mit demselben. . . . . . . . 554

Der Geisterlehre II. Abschnitt.

Von den Gründen alles dessen, was den Seelen und Geistern zukómmt.

Das I. Hauptstůck.

Von dem Wesen und der Natur der Seele. . . . . . 559

Das II. Hauptstůck.

Von der Empfindungs- und Einbildungskraft. . . . 565 
Das III. Hauptstůck.

Von dem Verstande und der Vernunft. . . . . . . . 571

Das IV. Hauptstůck.

Von den Begierden und dem Willen. .......576

Das V. Hauptstŭck.

Von der Vereinigung der Seele und des Leibes. . . . 581

Das VI. Hauptstůck.

Von der Unsterblichkeit der Seele. . . . . . . . . . . 588

Das VII. Hauptstůck.

Von dem Wesen des Geistes ủberhaupt. . . . . . . 591

Das VIII. Hauptstůck.

Von den Seelen der Thiere. . . . . . . . . . . . . 594

Der Geisterlehre III. Abschnitt.

Von dem unendlichen Geiste, oder von Gott.

Das I. Hauptstůck.

Beweis, daß ein Gott seyn můsse. . . . . . . . . 596

Das II. Hauptstůck.

Von den góttlichen Eigenschaften ůberhaupt. . . . . 601

Das III. Hauptstúck.

Von dem Wesen Gottes und dessen Unendlichkeit. 608 Das IV. Hauptstůck.

Von den Werken Gottes. . . . . . . . . . . . 616

Das V. Hauptstůck.

Von der Stadt Gottes, oder der Republik der Geister. 626 